\title{
Fertilidade e carbono orgânico do solo em sistemas agroflorestais de duas décadas compostos de castanheira, cupuaçuzeiro e pupunheira na Amazônia Ocidental
}

\author{
Soil fertility and organic carbon in two decades of agroforestry systems \\ composed of Brazil nut, cupuaçuzeiro and peach palm in the Western Amazon
}

\author{
Angelita Gude BUTZKE ${ }^{1}$; Tadário Kamel de OLIVEIRA²; Alex Elias Braga de PAULA ${ }^{3}$; \\ Sergio da Silva FIUZA ${ }^{4}$
}

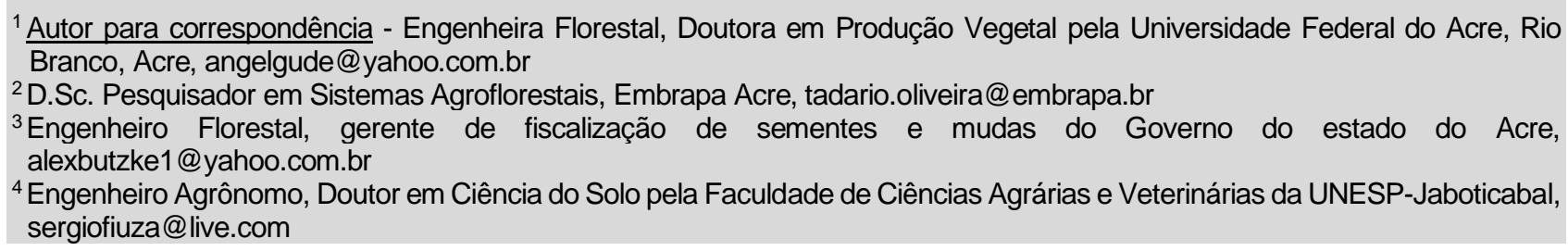

Recebido em: 24-12-2018; Aceito em: 20-04-2020

\begin{abstract}
Resumo
A Floresta Amazônica vem sendo substituída por diversos sistemas de uso, como pastagens e variados usos agrícolas. O objetivo deste estudo foi avaliar o efeito da implantação do sistema agroflorestal (SAF) na Amazônia sobre atributos químicos do solo em três SAFs, compostos por castanheira, cupuaçuzeiro e pupunheira, sendo o SAF A analisado aos 4 e 19 anos, o SAF B aos 7 e 22 anos, e o SAF C aos 6 e 21 anos após a implantação. Foram avaliados os teores de $\mathrm{P}, \mathrm{Ca}^{2+}, \mathrm{Mg}^{2+}, \mathrm{K}^{+}, \mathrm{H}+\mathrm{Al}, \mathrm{pH}, \mathrm{SB}, \mathrm{CTC}$, V\% e carbono orgânico total. O delineamento experimental foi em blocos ao acaso, em esquema de parcelas subdivididas, com dois tempos, constituídos da primeira e segunda coletas nas parcelas e três profundidades, sendo de 0-20, 20-40 e 40-60 cm nas subparcelas, totalizando seis tratamentos e 24 repetições, analisados independentemente nos três SAFs. Os resultados foram submetidos à análise de variância e as médias foram comparadas pelo teste de Tukey a 5\%. Após duas décadas do estabelecimento destes SAFs, houve perda de fertilidade e de carbono orgânico total do solo, além de que a maior disponibilidade de nutrientes e quantidade de matéria orgânica ficou concentrada na camada superficial de 0 a $20 \mathrm{~cm}$ de profundidade. Os resultados indicam que são necessárias medidas de reposição de nutrientes e correção da acidez em profundidade ou, dependendo dos objetivos produtivos destes sistemas, sua implantação seja realizada com maior diversificação e complexidade de espécies de exploração e de serviços ecossistêmicos.
\end{abstract}

Palavras-chave adicionais: indicadores de qualidade do solo; Rondônia; sustentabilidade.

\begin{abstract}
The Amazon rainforest has been replaced by several use systems, such as pastures and varied agricultural uses. The objective of this study was to evaluate the effect of the implantation of the agroforestry system (AS) in the Amazon on chemical attributes of the soil in three AS, composed of Brazil nut, cupuaçuzeiro and peach palm, being AS A analyzed at 4 and 19 years old, AS B at 7 and 22 years, and AS C at 6 and 21 years after implantation. The levels of $\mathrm{P}, \mathrm{Ca} 2+, \mathrm{Mg} 2+, \mathrm{K}+, \mathrm{H}+\mathrm{Al}, \mathrm{pH}, \mathrm{SB}, \mathrm{CTC}, \mathrm{V} \%$ and total organic carbon were evaluated. The experimental design was in randomized blocks, in a split plot scheme, with two stages, consisting of the first and second collections in the plots and three depths, being 0-20, 20-40 and 40-60 cm in the subplots, totaling six treatments and 24 repetitions, independently analyzed in the three SAFs. The results were subjected to analysis of variance and the means were compared using the Tukey test at 5\%. Two decades after the establishment of these AS, there was a loss of fertility and total organic carbon in the soil, in addition to the fact that the greater availability of nutrients and the amount of organic matter was concentrated in the superficial layer from $0-20 \mathrm{~cm}$ deep. The results indicate that nutrient replacement measures and in-depth acidity correction are necessary, or, depending on the productive objectives of these systems, their implementation should be carried out with greater diversification and complexity of species of exploration and ecosystem services.
\end{abstract}

Additional keywords: Rondônia; soil quality indicators; sustainability. 


\section{Introdução}

A ocupação desordenada na Amazônia brasileira colaborou com o incremento da taxa anual de desmatamento de suas florestas para a implantação, principalmente, de pastagens e de agricultura de baixa tecnificação, contribuindo significativamente em mudanças nos seus fluxos e ciclos naturais e atribuindo atualmente a esta região uma característica típica de uso da terra na região: o predomínio da pecuária de corte como principal uso. Mediante esta ocupação, a agricultura itinerante que se baseia no corte e queima da floresta para o estabelecimento das culturas sobre as cinzas, entre troncos e galhos parcialmente queimados, passou a ser a principal forma de agricultura na Amazônia e em especial no estado do Acre.

Com a retirada da cobertura vegetal original para implantação de culturas, aliadas às práticas de manejo inadequadas, dentre outros, resulta em danos ambientais como a perda de biodiversidade, empobrecimento dos solos e rompimento do equilíbrio entre o solo e o meio, modificando seus atributos físicos, químicos e biológicos, limitando sua utilização agrícola e tornando-o mais suscetível à degradação e erosão hídrica (Caetano et al., 2013; Moline \& Coutinho, 2015) e, sobretudo, lança na atmosfera grande quantidade de gases de efeito estufa, em especial o dióxido de carbono com as queimadas (Redin et al., 2011).

A utilização inadequada do solo ocasiona a degradação das características indicadoras de qualidade do solo (físicas, químicas e biológicas) tais como, a desestruturação e compactação, perdas de solo e nutrientes através da erosão e lixiviação, redução da fertilidade, oxidação acelerada da matéria orgânica, perdas de carbono $(C)$, nitrogênio $(N)$, fósforo $(P)$ e dos reservatórios de nutrientes associados a matéria orgânica e a diminuição da quantidade e diversidade de organismos do solo (Leite et al., 2010; Sá et al., 2010).

Isso reflete na redução da qualidade do solo e a interrupção da continuidade dos seus processos biológicos, que são responsáveis pela ciclagem e mineralização dos nutrientes orgânicos para a nutrição das plantas, proporcionando uma queda acentuada na produção vegetal (Limaet al., 2011).Na Amazônia Sul Ocidental, onde as taxas de desmatamento foram em menor escala quando comparadas com as demais regiões da Amazônia brasileira, essa típica agricultura itinerante ainda se faz presente, deixando um grande percentual de áreas degradadas com precária aptidão para implantação de determinados tipos culturas ou manejo de uso da terra.

No Acre, essa realidade permite indicar a utilização de sistemas agroflorestais - SAF's nas diversas modalidades, visando ocupar áreas já desmatadas e ao mesmo tempo gerar emprego e renda aos pequenos e médios produtores. Neste sentido Fearnside (1989) assegura que os sistemas agroflorestais devem ser encorajados apenas como uso da terra em áreas já desmatadas, ou severamente perturbadas, não como uma substituição de ecossistemas florestais naturais.
Por apresentarem similaridade com a floresta, os sistemas agroflorestais são opções atrativas para o uso do solo na Amazônia, constituindo uma alternativa sustentável (Oliveira et al., 2017) com possibilidade de auxiliar na redução do desmatamento uma vez que rompe com o ciclo da agricultura migratória, a qual, em função de períodos de pousio curtos para a recuperação dos solos, aumenta a pressão sobre as áreas de floresta primária (Oliveira et al., 2010).

Os sistemas agroflorestais têm maior potencial de sequestro de $\mathrm{C}$ e $\mathrm{N}$ em comparação com os locais adjacentes de monocultura (Chen et al., 2017), sendo a proporção do estoque de carbono orgânico nas camadas do subsolo maior em sistema agroflorestal do que em monocultivo (Guo et al., 2020). O acúmulo de carbono no solo é atribuído principalmente ao aumento da biomassa de resíduos das árvores e aos exsudatos radiculares, menos distúrbios humanos e mecanismos de proteção de carbono orgânico do solo submetido a alta atividade e quantidade microbiana (Liao \& Boutton, 2008)

As perdas de culturas causadas por pragas e doenças diminuem a renda e ameaçam os meios de subsistência de milhares de famílias em todo o mundo. Um bom exemplo da magnitude desses impactos são as enormes perdas de safras experimentados por cafeicultores da América Central devido à ferrugem da folha de café. Os cafeicultores precisam implantar agroecossistemas capazes de regular os impactos negativos de pragas e doenças e, ao mesmo tempo, proporcionar outros serviços ecossistêmicos dos quais dependem suas famílias e a sociedade (Cerda et al., 2020).

Neste contexto, sendo o solo um recurso natural complexo e dinâmico, o uso de indicadores da sua qualidade é essencial para avaliação e entendimento da funcionalidade e sustentabilidade de solos em diferentes condições de uso. No entanto, em sistemas agroflorestais esta abordagem ainda é carente de estudos, necessitando de pesquisas direcionadas na dinâmica dos atributos químicos, físicos e biológicos em função da utilização destes. Portanto, objetivo deste estudo foi avaliar o efeito da implantação do sistema agroflorestal na Amazônia sobre atributos químicos do solo em três áreas, sendo a primeira analisada aos $4 \mathrm{e}$ 19 anos, a segunda aos 7 e 22 anos, e a terceira aos 6 e 21 anos após a implantação.

\section{Material e métodos}

O estudo foi conduzido na Amazônia SulOcidental, nos limites entre os Estados do Acre e de Rondônia, com as áreas situadas em propriedades rurais no Distrito de Nova Califórnia, Município de Porto Velho, Rondônia. As áreas têm como rede viária principal a Rodovia Federal BR 364. Nesta região o clima predominante segundo a classificação de Köppen, é do tipo Aw, equatorial quente e úmido, caracterizado por altas temperaturas (com temperatura média anual do ar em torno de $25^{\circ} \mathrm{C}$ ) e elevados índi- 
ces de precipitação pluviométrica anual variando entre 1.400 a 2.500 mm, com estação seca bem acentuada nos meses de junho, julho e agosto (Sedam, 2012).

\section{Caracterização das Áreas avaliadas}

As áreas tiveram os Sistemas Agroflorestais implantados nos seguintes anos: SAF A em 1995, SAF B em 1992 e SAF C em 1993. No ano de1999, portanto 4, 7 e 6 anos após a implantação dos SAFs, respectivamente, foram realizadas coletas para análises químicas dos solos, ocasião em que as áreas de coletas foram georreferenciadas, o que permitiu o retorno aos locais para realização da segunda amostragem após 15 anos, em 2014.

\section{SAF A - Sistema Agroflorestal implantado em 1995 e} avaliado aos 4 e 19 anos

Área de estudo localizada no Ramal Pioneiro, km 04, no Distrito de Nova Califórnia, município de Porto Velho, Rondônia. O solo foi classificado como
LATOSSOLO AMARELO Distrófico argissólico, textura argilosa no horizonte $A$, a muito argilosa no horizonte $B$; epieutrófico com presença de concreções lateríticas; A fraco, relevo plano, altitude de $192 \mathrm{~m}$ e coordenadas UTM (DATUM SAD 69 Zona 19L) $\mathrm{x}=759.413 \mathrm{e}$ $\mathrm{y}=8.917 .254$ possuindo um total de 17 hectares de SAF's, o que representa $14,5 \%$ do total da propriedade, sendo esta área componente da sua reserva legal.

Nesta área, o SAF foi implantado no ano de 1995 com o arranjo demostrado na Figura 1, sendo na linha principal a presença de castanheira (Bertholletia excelsa) intercalada com cupuaçuzeiro (Theobroma grandiflorum) e nas entrelinhas, uma linha de pupunheira (Bactris gasipaes) pura e outra de pupunheira com cupuaçuzeiro. O espaçamento foi de $14 \times 12 \mathrm{~m}$ para as castanheiras, $7 \times 4 \mathrm{~m}$ para as pupunheiras e $14 \times 8 \mathrm{~m}$ para os cupuaçuzeiros (Figura 1). Foi realizada adubação com esterco de gado de 10 a 12 litros por planta na época da implantação, sendo o SAF's conduzido até a presente data sem colheita rigorosa, permanecendo no solo do sistema a maior parte da produção.

\begin{tabular}{|c|c|c|c|c|c|}
\hline \multicolumn{2}{|c|}{7 m (Linha) } & \multirow[b]{2}{*}{$\Delta$} & \multirow[b]{2}{*}{$\because$} & \multirow[b]{2}{*}{$\Delta$} & \multirow[b]{2}{*}{$\because$} \\
\hline$\Delta$ & $\because$ & & & & \\
\hline$¥$ & $¥$ & $¥$ & $¥$ & $¥$ & $¥$ \\
\hline 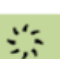 & $¥$ & 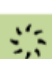 & $¥$ & 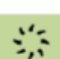 & $¥$ \\
\hline$\Delta$ & “" & $\Delta$ & ב־" & $\Delta$ & בַּ \\
\hline$¥$ & $¥$ & $¥$ & $¥$ & $¥$ & $¥$ \\
\hline “", & $¥$ & 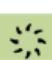 & $¥$ & 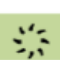 & $¥$ \\
\hline$\Delta$ & 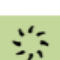 & $\Delta$ & 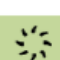 & $\Delta$ & 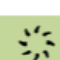 \\
\hline
\end{tabular}

\footnotetext{
Legenda: $\Delta=$ Castanheira $(14 \mathrm{~m} \times 12 \mathrm{~m})$

$\because=$ = Cupuaçuzeiro $(14 \mathrm{~m} \times 8 \mathrm{~m})$

$¥=$ Pupunheira $(7 \mathrm{~m} \times 4 \mathrm{~m})$
}

Figura 1 - Croqui do arranjo do Sistema Agroflorestal, SAF A, localizado no Ramal Pioneiro, km 04, no Distrito de Nova Califórnia, Porto Velho, Rondônia. Sketch of the arrangement of the Agroforestry System, SAF A, located at Ramal Pioneiro, km 04, in the District of Nova California, Porto Velho, Rondônia.

SAF B - Sistema Agroflorestal implantado em 1992 e avaliado aos 7 e 22 anos

Área de estudo localizada no Ramal Linha 5, km 05, no Distrito de Nova Califórnia, município de Porto Velho, Rondônia. O solo foi classificado em ARGISSOLO VERMELHO AMARELO Distrófico latossólico, textura média a argilosa, relevo local plano a suave ondulado, altitude de $152 \mathrm{~m}$ e coordenadas UTM (DATUM SAD 69 Zona 19L) $\mathrm{x}=774.238$ e $\mathrm{y}=8.921 .988$ possuindo um total de 14 hectares de
SAF's, o que representa $14 \%$ do total da propriedade, sendo esta área componente da sua reserva legal.

Nesta área, o sistema agroflorestal foi implantado no ano de 1992, sendo na linha principal a presença de castanheira (Bertholletia excelsa) intercalada com cupuaçuzeiro (Theobroma grandiflorum) e na entrelinha, uma linha de pupunheira (Bactris gasipaes) com cupuaçuzeiro. O espaçamento foi de $14 \times 8 \mathrm{~m}$ para todas as culturas (Figura 2). 


\begin{tabular}{|c|c|c|c|c|c|}
\hline \multicolumn{2}{|c|}{7 m (Linha) } & \multirow[b]{2}{*}{$\Delta$} & \multirow[b]{2}{*}{$\because$} & \multirow[b]{2}{*}{$\Delta$} & \multirow[b]{2}{*}{;“; } \\
\hline$\Delta$ & 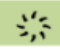 & & & & \\
\hline$\because$ & $¥$ & $\because$, & $¥$ & $\because$ & $¥$ \\
\hline$\Delta$ & 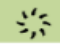 & $\Delta$ & $\because$ & $\Delta$ & 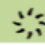 \\
\hline 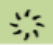 & $¥$ & $\because$ & $¥$ & “; & $¥$ \\
\hline$\Delta$ & $\because ;$ & $\Delta$ & 茫 & $\Delta$ & 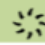 \\
\hline$\because$ & $¥$ & 棌 & $¥$ & $\because$ & $¥$ \\
\hline$\Delta$ & 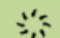 & $\Delta$ & (“) & $\Delta$ & 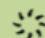 \\
\hline
\end{tabular}

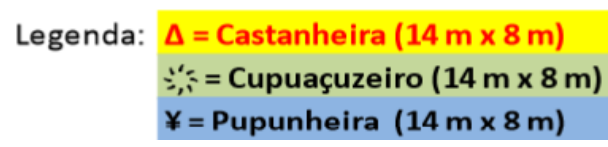

Figura 2 - Croqui do arranjo do Sistema Agroflorestal, SAF B, localizado no Ramal Linha 5, km 05, no Distrito de Nova Califórnia, Porto Velho, Rondônia. Sketch of the arrangement of the Agroforestry System, SAF B, located at Branch Line 5, km 05, in the District of Nova California, Porto Velho, Rondônia.

Foram utilizadas calagem e adubação foliar (dosagem não especificada) no ano de 1998, sendo o sistema agroflorestal conduzido até a presente data com colheita habitual constante, exceto colheita dos frutos da pupunheira devido à altura das árvores. Foram introduzidas ao sistema novas espécies florestais como Cedro (Cedrela odorata), Andiroba (Carapa guianensis), Mogno (Swietenia macrophylla), Copaíba (Copaifera sp.), Cumaru Cetim (Apuleia moralis), Ipê (Tabebuia sp.) e Paricá (Schizolobium amazonicum) sem espaçamento planejado a partir do ano de 2010. Atualmente o sistema agroflorestal é uma das principais fontes de renda do proprietário.

\section{SAF C - Sistema Agroflorestal implantado em 1993 e} avaliado aos 6 e 21 anos

Área de estudo localizada na BR-364, km 1080, no Distrito de Nova Califórnia, município de Porto Velho, Rondônia. O solo foi classificado em LATOSSOLO VERMELHO Distrófico argissólico, textura muito argilosa, área pequena localizada no topo da paisagem, relevo local plano em relevo regional suave ondulado a ondulado, altitude de $188 \mathrm{~m}$ e coordenadas UTM (DATUM SAD 69 zona 19L) $x=767.683$ e $y=8.918 .470$ possuindo um total de 3 hectares de SAF's, o que representa $3 \%$ do total da propriedade, sendo esta área componente da sua reserva legal. Nesta área, o sistema agroflorestal foi implantado no ano de 1993, sendo na linha principal a presença de castanheira (Bertholletia excelsa) intercalada com cupuaçuzeiro (Theobroma grandiflorum) e nas entreli- nhas, uma linha de pupunheira (Bactris gasipaes) com cupuaçuzeiro e outra com apenas cupuaçuzeiro. $\mathrm{O}$ espaçamento foi de $16 \times 12 \mathrm{~m}$ para as castanheiras, $4 \times 8 \mathrm{~m}$ para os cupuaçuzeiros e $16 \times 8 \mathrm{~m}$ para as pupunheiras (Figura 3). Foi utilizado adubo orgânico (sem dosagem especificada) no ano de implantação, sendo o sistema agroflorestal conduzido até a presente data com colheita habitual constante, exceto colheita dos frutos da pupunheira devido à altura das árvores. Atualmente o Sistema agroflorestal é uma das principais fontes de renda do proprietário.

\section{Descrição da coleta de dados nas áreas dos SAF's}

As coletas de solo foram realizadas nos meses de maio a junho de 2014, sendo que para cada área, foram obtidas informações do histórico de uso com os proprietários atuais e antigos e coletadas amostras de solo. Considerando o ponto georrefenciado como o centro e um círculo com 100 m de diâmetro, foram abertas trincheiras para serem coletadas amostras de solo, em camadas nas profundidades de 0-20, 20-40, 40-60 cm para análise de fertilidade, distribuídas nos eixos do cruzamento com os pontos cardeais $(\mathrm{N}, \mathrm{S}, \mathrm{L}$, O), sendo estes, os pontos de amostragens seguindo metodologia descrita por Amaral et al. (2001).

Cada uma das 4 trincheiras abertas por área para coleta de solo foi considerada como um bloco georrefenciado com GPS Garmim MAP 76CSx. Após as coletas as amostras foram encaminhadas ao Laboratório de Solos da Embrapa Acre onde se procedeu às análises. 


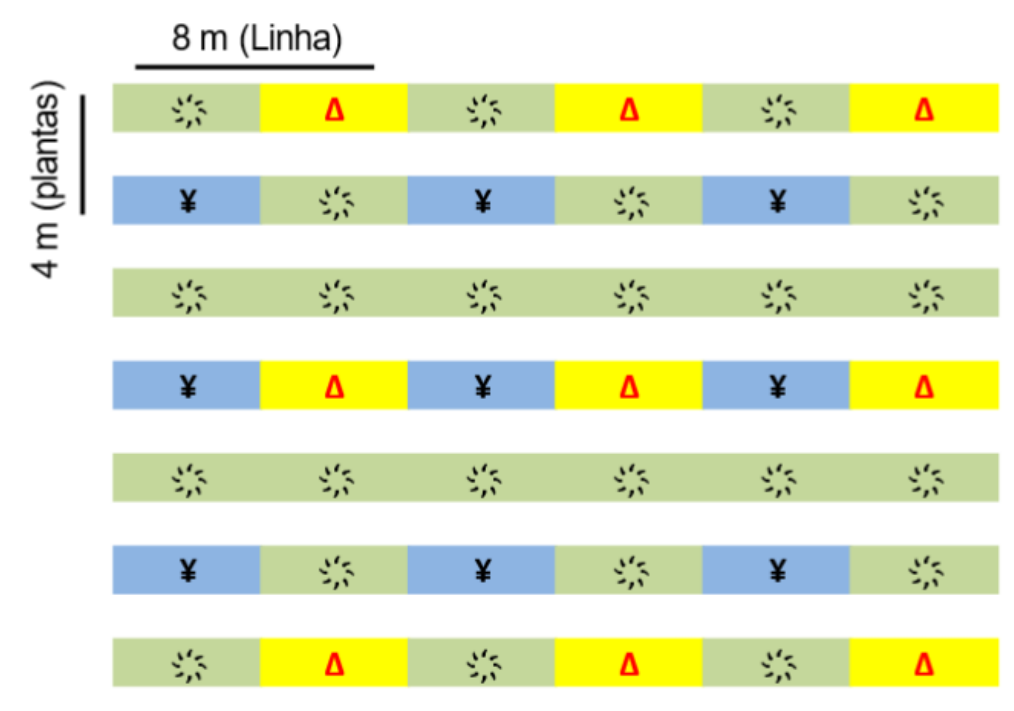

Legenda: $\Delta=$ Castanheira $(16 \mathrm{~m} \times 12 \mathrm{~m})$

$\because,:=$ Cupuaçuzeiro $(4 \mathrm{~m} \times 8 \mathrm{~m})$

$P=$ Pupunheira (16 $\mathrm{m} \times 8 \mathrm{~m})$

Figura 3 - Croqui do Sistema Agroflorestal, SAF C, localizado na BR-364, km 1080, no Distrito de Nova Califórnia, Porto Velho, Rondônia. Sketch of the Agroforestry System, SAF C, located at BR-364, km 1080, in the District of Nova California, Porto Velho, Rondônia.

\section{Análises laboratoriais}

Foram analisados, conforme metodologia da

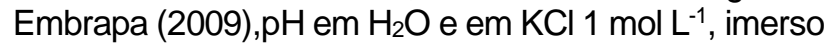
em suspensão solo/água na proporção de 1:2,5; cálcio, magnésio e alumínio trocáveis, foram extraídos por $\mathrm{KCl}$ $1 \mathrm{~mol} \mathrm{~L}^{-1}$ e quantificados por espectrofotometria de absorção atômica; fósforo, potássio e sódio trocáveis foram extraídos com solução duplo-ácida $\mathrm{HCl}$ $0,05 \mathrm{~mol} \mathrm{~L}^{-1}+\mathrm{H}_{2} \mathrm{SO}_{4}$ 0,0125 mol L-1 (Mehlich 1), sendo o fósforo disponível determinado por espectrofotometria, o potássio e o sódio trocáveis pelo método de fotômetro de chama. A acidez potencial $(\mathrm{H}+\mathrm{Al})$ foi extraída com solução de acetato de cálcio $0,5 \mathrm{~mol} \mathrm{~L}^{-1}$ ajustada a pH 7,0, sendo determinada por titulação com solução de $\mathrm{NaOH} 0,025 \mathrm{~mol} \mathrm{~L}^{-1}$.

O carbono orgânico total foi determinado por meio do processo de oxidação da matéria orgânica, por via úmida, com dicromato de potássio $0,1667 \mathrm{~mol} \mathrm{~L}^{-1}$ sem aquecimento (Walkley\& Black, 1934). A titulação foi realizada com sulfato ferroso amoniacal $0,1 \mathrm{~mol} \mathrm{~L}^{-1}$ (Defelipo\& Ribeiro, 1997).

Para cada repetição também foram calculadas a soma de bases, capacidade de troca catiônica e saturação por bases.

\section{Delineamento experimental e análises estatísticas}

O delineamento experimental foi em blocos ao acaso, em esquema de parcelas subdivididas em dois tempos (primeira coleta e segunda coleta) nas parcelas e três profundidades $(0-20,20-40$ e $40-60 \mathrm{~cm})$ nas subparcelas, totalizando seis tratamentos, com quatro repetições e 24 amostras em cada SAF.

As análises foram realizadas para cada área isoladamente, não havendo comparação de resultados entre elas, mas somente comparação de resultados das análises químicas de solos provenientes das coletas realizadas em 1999 (primeira análise) e 2014 (segunda análise) dentro da mesma área, o que corresponde a um tempo de uso de quinze anos.

Os dados foram submetidos à análise estatística, após serem verificados os pressupostos da análise de variância - a normalidade dos resíduos pelo teste de Shapiro \& Wilk (1965) e a homogeneidade das variâncias pelo teste de Bartlett (1937). As variáveis que necessitaram de transformação para atender aos pressupostos da análise de variância em duas das três áreas foram: SAF A - H+Al e carbono orgânico $(\sqrt{ } \mathrm{x}), \mathrm{V} \%$ $(\cos x+1), K(\sqrt{ } x / x)$; SAF C, $H+A I(\sqrt{ } x / x)$. Posteriormente efetuou-se a análise de variância dos dados originais e/ou transformados e verificados pelo teste $\mathrm{F}$ a existência ou não de diferença significativa $(p<0,05)$ entre os tratamentos.

Para as variáveis que apresentaram diferença significativa com relação ao tempo (antes e depois), profundidades $(0-20,20-40$ e $40-60 \mathrm{~cm})$ e sua interação, foi aplicado o teste de Tukey (Tukey,1949) a 5\% de probabilidade. Para o processamento dos dados foi utilizado o programa estatístico Assistat 7.7 (Silva, 2013). 


\section{Resultados e discussão}

O efeito no solo em função do tempo de implantação dos sistemas agroflorestais- SAF A, SAF B e SAF C, são apresentados na Tabela 1. Não houve diferenças significativas para a maioria das variáveis analisadas na comparação do SAF A aos 4 e 19 anos, exceto quanto ao fósforo disponível que diminuiu de $0,92 \mathrm{mg} \mathrm{dm}^{-3}$ para $0,64 \mathrm{mg} \mathrm{dm}^{-3}$.

Tabela 1 - Atributos químicos de fertilidade do solo avaliados no período inicial e atual de implantação de três sistemas agroflorestais (SAFs) compostos por castanheira, cupuaçuzeiro e pupunheira, no distrito de Nova Califórnia - RO. Chemical attributes of soil fertility evaluated in the initial and current period of implantation of three agroforestry systems (SAFs) composed of Brazil nut, cupuaçuzeiro and peach palm, in the district of Nova Califórnia - RO.

\begin{tabular}{|c|c|c|c|c|c|c|c|c|c|c|}
\hline \multirow{2}{*}{$\begin{array}{l}\text { Sistemas } \\
\text { Agroflorestais }\end{array}$} & $\mathrm{Ca}$ & $\mathrm{Mg}$ & $\mathrm{K}$ & SB & $\mathrm{H}+\mathrm{Al}$ & CTC & $\mathrm{V}$ & $\mathrm{CO}$ & $\mathrm{P}$ & $\mathrm{pH}$ \\
\hline & \multicolumn{6}{|c|}{ 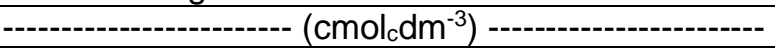 } & $(\%)$ & $\left(\mathrm{g} \mathrm{kg}^{-1}\right)$ & $\left(\mathrm{mg} \mathrm{dm}^{-3}\right)$ & $\mathrm{H}_{2} \mathrm{O}$ \\
\hline SAF A & & & & & & & & & & \\
\hline 4 anos & $1,52 \mathrm{a}$ & $0,47 a$ & $0,25 a$ & $2,25 a$ & $4,70 a$ & $7,00 a$ & $30,00 a$ & $1,29 a$ & $0,92 a$ & $5,20 a$ \\
\hline $\begin{array}{l}19 \text { anos } \\
\text { SAF B }\end{array}$ & $1,63 a$ & $0,55 a$ & $0,12 \mathrm{a}$ & $2,30 a$ & $3,43 a$ & $5,76 a$ & & $0,96 a$ & $0,64 b$ & $5,05 a$ \\
\hline 7 an & $59 a$ & $0,72 a$ & $0,08 a$ & 1,40 & & 8,60 & & $0,97 a$ & $1,18 \mathrm{a}$ & $4,58 \mathrm{a}$ \\
\hline $\begin{array}{l}22 \text { anos } \\
\text { SAF C }\end{array}$ & $0,49 b$ & $0,20 \mathrm{~b}$ & $0,04 b$ & $0,74 b$ & $4,43 b$ & $5,18 b$ & & $0,87 a$ & $0,90 a$ & $4,56 \mathrm{a}$ \\
\hline 6 anos & $40 a$ & $0,40 a$ & $0,08 a$ & $2,55 a$ & $7,97 a$ & $10,53 a$ & $22,22 a$ & $1,22 \mathrm{a}$ & $1,33 a$ & $4,29 a$ \\
\hline 21 anos & $0,55 b$ & $0,16 \mathrm{~b}$ & $0,02 b$ & $0,74 b$ & $4,85 b$ & $5,60 \mathrm{~b}$ & $9,19 a$ & $0,95 b$ & $1,61 \mathrm{a}$ & $4,21 \mathrm{a}$ \\
\hline
\end{tabular}

Médias seguidas da mesma letra na coluna não diferem estatisticamente entre si pelo teste $\mathrm{F}$ ao nível de $5 \%$ de probabilidade.

Houve perdas significativas nos SAF B e SAF $\mathrm{C}$, considerando o tempo entre as avaliações, nos teores de cálcio, magnésio, potássio, soma e saturação por bases; por outro lado, nestes sistemas agroflorestais, houve redução da acidez potencial $(\mathrm{H}+\mathrm{Al})$,no entanto, esses solos continuam com acidez elevada, necessitando de correção. Houve tendência de redução do carbono orgânico total nos três sistemas agroflorestais avaliados, mas, de forma significativa $(p<0,05)$ apenas no SAF C, de $1,22 \mathrm{~g} \mathrm{~kg}^{-1}$ para $0,95 \mathrm{~g} \mathrm{~kg}^{-1}$.
Estes resultados indicam que há desequilíbrio nos sistemas agroflorestais avaliados entre as taxas de exportação de nutrientes pelas colheitas dos frutos e a ciclagem de nutrientes proveniente da biomassa, situação que poderá reduzir a produtividade e sustentabilidade do sistema.

Os resultados de fertilidade, considerando as avaliações feitas nas profundidades de $0-20 \mathrm{~cm}, 20-40 \mathrm{~cm}$ e 40-60 cm dos três sistemas agroflorestais são apresentados na Tabela 2.

Tabela 2 - Atributos químicos de fertilidade do solo de três sistemas agroflorestais (SAFs) compostos por castanheira, cupuaçuzeiro e pupunheira, avaliados no período inicial e atual de implantação, nas profundidades de 0-20 cm, 20-40 cm e 40-60 cm, no distrito de Nova Califórnia - RO. Chemical attributes of soil fertility of three agroforestry systems (SAFs) composed of Brazil nut, cupuaçuzeiro and peach palm, evaluated in the initial and current period of implantation, in the depths of $0-20 \mathrm{~cm}, 20-40 \mathrm{~cm}$ and $40-60 \mathrm{~cm}$, in the district from California - RO.

\begin{tabular}{|c|c|c|c|c|c|c|c|}
\hline $\begin{array}{c}\text { SAF A } \\
\text { Profundidade }(\mathrm{cm})\end{array}$ & \multicolumn{3}{|c|}{$\mathrm{Ca}$} & \multicolumn{2}{|c|}{ SB } & \multicolumn{2}{|c|}{$\begin{array}{c}\mathrm{P} \\
\left(\mathrm{mg} \mathrm{dm}^{-3}\right)\end{array}$} \\
\hline $\begin{array}{r}0-20 \\
20-40 \\
40-60\end{array}$ & \multicolumn{2}{|c|}{$\begin{array}{l}1,42 b \\
0.71 b\end{array}$} & & \multicolumn{2}{|c|}{$\begin{array}{l}1,98 b \\
1,13 b\end{array}$} & \multicolumn{2}{|c|}{$\begin{array}{l}0,76 \mathrm{ab} \\
0.61 \mathrm{~b}\end{array}$} \\
\hline $\begin{array}{c}\text { SAF B } \\
\text { Profundidade }(\mathrm{cm})\end{array}$ & Ca & $\mathrm{Mg}$ & $\begin{array}{c}\mathrm{K} \\
\mathrm{cmol}_{\mathrm{c}} \mathrm{dn} \\
\end{array}$ & SB & CTC & $\begin{array}{c}\mathrm{CO} \\
\left(\mathrm{g} \mathrm{kg}^{-1}\right)\end{array}$ & $\begin{array}{c}\mathrm{P} \\
\left(\mathrm{mg} \mathrm{dm}^{-3}\right)\end{array}$ \\
\hline $\begin{array}{r}0-20 \\
20-40 \\
40-60\end{array}$ & $\begin{array}{l}1,09 \mathrm{a} \\
0,33 \mathrm{~b} \\
0,22 \mathrm{~b}\end{array}$ & $\begin{array}{l}0,67 \mathrm{a} \\
0,38 \mathrm{~b} \\
0,34 \mathrm{~b}\end{array}$ & $\begin{array}{l}0,10 \mathrm{a} \\
0,05 \mathrm{~b} \\
0,04 \mathrm{~b}\end{array}$ & $\begin{array}{l}1,86 \mathrm{a} \\
0,76 \mathrm{~b} \\
0,60 \mathrm{~b}\end{array}$ & $\begin{array}{l}7,97 \mathrm{a} \\
6,52 \mathrm{~b} \\
6,20 \mathrm{~b}\end{array}$ & $\begin{array}{l}1,27 \mathrm{a} \\
0,83 \mathrm{~b} \\
0,68 \mathrm{~b}\end{array}$ & $\begin{array}{l}1,77 \mathrm{a} \\
0,76 \mathrm{~b} \\
0,60 \mathrm{~b}\end{array}$ \\
\hline $\begin{array}{c}\text { SAF C } \\
\text { Profundidade }(\mathrm{cm})\end{array}$ & 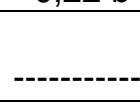 & & $\mathrm{cmol}_{\mathrm{c}} \mathrm{dm}$ & & CTC & $\begin{array}{c}\mathrm{V} \\
(\%)\end{array}$ & $\begin{array}{c}\mathrm{CO} \\
\left(\mathrm{g} \mathrm{kg}^{-1}\right)\end{array}$ \\
\hline $\begin{array}{r}0-20 \\
20-40 \\
40-60\end{array}$ & & & & & $\begin{array}{l}9,73 \mathrm{a} \\
6,93 \mathrm{~b} \\
7,53 \mathrm{~b}\end{array}$ & $\begin{array}{l}29,01 \mathrm{a} \\
8,79 \mathrm{~b} \\
10,40 \mathrm{~b}\end{array}$ & $\begin{array}{l}1,45 \mathrm{a} \\
0,85 \mathrm{~b} \\
0,96 \mathrm{~b}\end{array}$ \\
\hline
\end{tabular}

Médias seguidas da mesma letra na coluna não diferem estatisticamente entre si pelo teste $\mathrm{F}$ ao nível de $5 \%$ de probabilidade. 
A camada superficial do solo, na profundidade de $0-20 \mathrm{~cm}$, é a de maior fertilidade, concentrando os nutrientes: no SAF A, cálcio, soma de bases e fósforo; no SAF B, cálcio, magnésio, potássio, soma de bases, CTC, carbono orgânico e fósforo; e no SAF C, cálcio, soma de bases, CTV, saturação por bases e carbono orgânico.

Na mesma região deste estudo, os maiores teores de nutrientes na classe de LATOSSOLO VERMELHO encontram-se nas camadas superficiais (Amaral et al., 2000),desta forma, estes autores recomendaram que no preparo para o plantio seja evitado o revolvimento da camada superficial, por apresentarem maior conteúdo de matéria orgânica, essenciais ao desenvolvimento das culturas nos sistemas agroflorestais.

Os sistemas agroflorestais avaliados foram implantados com espécies destinadas a exploração dos frutos- castanha (Bertholletia excelsa), cupuaçu (Theobroma grandiflorume) pupunha (Bactris gasipaes). O SAF A, que manteve a maioria dos indicadores avaliados inalterados, teve pressão de exploração baixa, com menor frequência de coleta dos frutos.

Sistemas agroflorestais devem incluir o maior número possível de espécies vegetais, proporcionar e manter altos níveis de matéria orgânica e alta diversidade dos organismos do solo, sistema radicular com distribuição estratificada, mantendo o solo mais produtivo e eficiente na utilização de água, luz e ciclagem de nutrientes.

Neste estudo, não foi avaliada a produtividade do sistema no tempo, que pode estar diminuindo em função da perda de nutrientes. Os resultados indicam que os objetivos produtivos destes sistemas seriam compatíveis com a necessidade de adubações externas suplementares, ou, caso contrário, careceram de maior diversificação com espécies que proporcionam serviços ecossistêmicos.

Os resultados evidenciam que o desafio vai além do planejamento dos objetivos de produção, da composição e complexidade de espécies para exploração e para serviços ecossistêmicos, exigindo outras estratégias de adubação e correção em profundidade, como a aplicação de gesso, que podem ser necessárias nestes sistemas agroflorestais.

Levantamentos de solos no projeto de Reflorestamento Econômico Consorciado Adensado (RECA), localizado na mesma região onde foram realizados este estudo, constataram que a classe LATOSSOLO VERMELHO apresenta reação variando de extremamente a fortemente ácida, com valores de $\mathrm{pH}$ na ordem de 3,9 a 5,3, sendo recomendado pelos autores a aplicação de calcário para elevar os valores de $\mathrm{pH}$ dos horizontes superficiais, indispensáveis para a maioria das culturas (Amaral et al., 2000).

Os resultados obtidos diferem de estudos que encontraram aumento na disponibilidade de nutrientes ao longo do tempo, com enriquecimento das camadas superficiais do solo, devido ao constante aporte de biomassa em sistemas agroflorestais (Ribeiro et al., 2019; Iwata et al., 2012).Sistemas agroflorestais de seis anos de idade com pupunheira e cupuaçuzeiro, os resultados de cálcio, magnésio, CTC e pH do solo foram maiores que na floresta e pastagem adjacentes (McGrath et al., 2001).

Apesar dos resultados controversos na literatura, a dinâmica de nutrientes na solução do solo pode não ser suficiente para manter ou melhorar as condições químicas do solo em função do tempo de implantação de sistemas agroflorestais, sendo, portanto, recomendado o enriquecimento dos arranjos por meio de práticas corretivas, sejam elas de forma química ou orgânica (Silva et al., 2011). A diminuição nos teores de bases trocáveis também pode ser entendida pela característica dos sistemas agroflorestais em promover um ambiente mais equilibrado, provavelmente em função da imobilização temporária na biomassa viva e/ou exportação via colheita dos frutos (Campos, 2015).

Teores de potássio foram reduzidos a níveis extremamente baixos em sistemas agroflorestais do projeto de Reflorestamento Econômico Consorciado Adensado (RECA) no município de Nova Califórnia, Rondônia, compostos de cupuaçuzeiro e pupunheira para exploração de palmito, devido às exportações de nutrientes por extrações consecutivas das frutas, sendo o potássio um dos nutrientes mais importantes na produção dessas frutíferas (Ayres \& Alfaia, 2007; Luizão et al., 2009).

A exportação de nutrientes via produção é a principal causa da diminuição da fertilidade do solo em sistemas agroflorestais avaliados na Amazônia SulOcidental (Luizão et al., 2009). A seleção de espécies e o planejamento do manejo conseguem produzir um regime de nutrientes adequado para manter ou melhorar a fertilidade do solo na Amazônia brasileira (Fernandes et al., 1997).

Embora nos sistemas agroflorestais a ciclagem biogeoquímica contribua para aumentar a disponibilidade de vários nutrientes nas primeiras camadas dos solos, Silva et al. (2011) recomendam a implantação de práticas corretivas e de adubação orgânica ou química, objetivando suprir o sistema em níveis adequados de nutrientes, como forma de repor os nutrientes nos sistemas agroflorestais do tipo multiestratificados, pois nestes, a disponibilidade de nutrientes é comprometida pela remoção constante via colheitas, geralmente maiores que a entrada natural.

Considerando o alto custo dos fertilizantes minerais e as características dos solos da região que favorecem a lixiviação de nutrientes, uma alternativa recomendação feita aos agricultores da mesma região desta pesquisa, foi a de substituir a queima das cascas da fruta cacau, ricas em potássio, por sua trituração e aplicação em superfície nos solos dos sistemas agroflorestais (Luizão et al., 2009).

Nos três sistemas agroflorestais avaliados houve perda de carbono orgânico do solo, mas de forma significativa apenas no SAF C. Fatores como tamanho e estabilidade de agregados, percentual de 
argila, quantidade e estratificação de raízes finas e hifas de fungos no solo, estão diretamente relacionadas com a dinâmica da matéria orgânica e ciclagem de nutrientes. Como a formação de agregados está associada ao aumento do armazenamento de carbono, a diminuição do conteúdo de carbono orgânico pode corresponder a diminuição do tamanho e perda de estabilidade dos macroagregados do solo.

Em sistemas agroflorestais à base de seringueira, Hevea brasiliensis, avaliados na China, houve aumento significativo do carbono orgânico total do solo (COT), do conteúdo de nitrogênio e formação de macroagregados nos SAFs formados por $H$. brasiliensis/ IT. cacau; $H$. brasiliensis/F. macrophylla; e H. brasiliensis/D. cochinchinensis, em comparação com o tratamento da monocultura de seringueira (Chenet al., 2017). Porém, a exceção ocorreu no SAF formado por $H$. brasiliensis/C. arábica, em que os autores observaram redução na proporção de macroagregados do solo, cuja formação é associada às raízes finas; de acordo com estes autores, apenas $33 \%$ das raízes finas do café estavam localizadas nos $10 \mathrm{~cm}$ superiores do solo e $73 \%$ estavam localizadas nos $30 \mathrm{~cm}$ de mais profundos.

A influência de árvores jovens e maduras, de 22 anos de Quercusilex, consorciada com ervilhaca e aveia, sob pastejo de ovelhas, aumentou a concentração e o conteúdo de carbono orgânico do solo, porém, os sistemas apresentaram distribuição semelhante de carbono orgânico do solo entre suas diferentes frações, sendo a fração desprotegida a dominante, seguida pelas frações protegidas física e quimicamente (Reyna-Bowen et al., 2020). De acordo com os autores, a distribuição do carbono orgânico do solo nas frações física e quimicamente desprotegidas foi fortemente correlacionada com a concentração total de carbono orgânico no solo, indicando a resposta rápida dessas frações ao manejo, mas não apresentou correlação com a fração bioquimicamente protegida, sugerindo que há alta resiliência às mudanças no estoque de carbono.

Estudo realizado no sul da França, em uma parcela agroflorestal de 18 anos, onde nozes híbridas (Juglans regia $\times$ Nigra L.) foram consorciadas com trigo (Triticum turgidum L. subsp. durum), e em uma parcela de controle agrícola adjacente, onde o trigo foi o único cultivo, mostraram que a maior parte do armazenamento adicional de carbono orgânico ao solo foi encontrada em frações orgânicas grosseiras (50-200 e

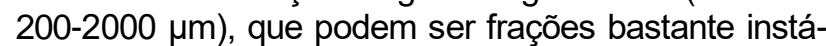
veis (Cardinaelet al., 2015).O estudo demonstrou o potencial dos sistemas agroflorestais em condições mediterrâneas para armazenar carbono orgânico no solo, porém questionou a estabilidade desse armazenamento.

Sistemas agroflorestais mais promissores de café, diferindo em suas práticas de cultivo e nos objetivos de rentabilidade dos agricultores, visando reduzir perdas e ao mesmo tempo fornecer outros serviços ecossistêmicos foram identificados em Turrialba, Costa
Rica, por meio da quantificação das perdas primária e secundária de café - rendimento e perdas econômicas - e de indicadores de três outros serviços ecossistêmicos: fornecimento de produtos dos sistemas agroflorestais (bananas, outras frutas e madeira), manutenção da fertilidade do solo e sequestro de carbono, (Cerda et al., 2020).Dentre os sistemas recomendados, conforme o perfil dos agricultores, um sistema simples, dominado por árvores de serviço, com objetivo de alta produtividade de café, apresentaram alta dependência de adubações e controle químico; os sistemas de diversificação média, para produção aceitável de café, caracterizado pelo uso racional de fertilizantes e de controle químico, e sistemas altamente diversificadas, que incluem árvores de serviço, árvores de madeira, árvores de fruto e bananeiras, com produção aceitável e baixa entrada de adubações e de controle químico.

Sistemas Agroflorestais de cacau derivados de áreas de savana e de florestas em Camarões foram avaliados quanto aos atributos relacionados aseis serviços/desserviços ecossistêmicos - conservação de espécies, estoque de carbono, produção agrícola, ciclagem de nutrientes, qualidade e poluição do solo seguindo sua evolução por mais de 70 anos (Nijmeijer et al., 2019). Os resultados mostraram que os atributos de serviços estudados geralmente tendem a atingir níveis comparáveis a longo prazo, se afastando progressivamente do uso anterior da terra, com tempo necessário variando de 20 a 30 anos quanto a diversidade de espécies perenes, e mais de 70 anos para armazenamento de carbono e outros componentes de qualidade do solo. Em relação aos atributos avaliados, tanto o sistema derivado de floresta, quanto o da savana, pareciam influenciados pelo uso anterior da terra até15 e 30 anos, respectivamente, após seu estabelecimento, com relação à qualidade do solo, ciclagem de nutrientes e armazenamento de carbono.

Os efeitos da composição de espécies de árvores, arbustos e ervas nos nutrientes do solo, devido ao aumento da complexidade da estrutura da comunidade de plantas, foram medidos por meio das concentrações de $\mathrm{C}, \mathrm{N}, \mathrm{P}, \mathrm{K}, \mathrm{Ca}$ e $\mathrm{Mg}$, em seis profundidades em plantação de monocultura de seringueira, $H$. brasiliensis, e quatro sistemas agroflorestais de multiespécies de seringueiras e uma floresta tropical. À medida que a composição das espécies vegetais aumentou, o carbono e nitrogênio do solo aumentaram; porém, a competição entre as plantas, devido ao aumento da complexidade, diminuiu a concentração de nutrientes do solo, principalmente $\mathrm{P}, \mathrm{K}$ e Ca (Wu et al., 2020).

Sistemas agroflorestais na Uganda, com café arábica (Coffea arábica) e conilon (Coffea canéfora), foram avaliados quanto ao sequestro de carbono no solo, em relação aos seus monocultivos e em função da composição do sistema com árvores frutíferas e não frutíferas (Tumwebaze \& Byakagaba, 2016). Nos sistemas agroflorestais com café arábica, os sistemas compostos com árvores frutíferas e não frutíferas estocaram maior quantidade de carbono; enquanto nos 
sistemas agroflorestais com café conilon, os sistemas com apenas árvores não frutíferas foram os que tiveram maior estoque de carbono no solo. Independentemente do tipo de café, os sistemas agroflorestais sequestraram mais carbono que as monoculturas.

A quantificação do estoque de carbono em diferentes sistemas agroflorestais de cacau (Theobroma cacao L.), floresta nativa e pastagem, avaliados até $100 \mathrm{~cm}$ de profundidade, localizados na Bahia, concluiu que o sistema com cacau e seringueira de 4 anos de idade foi o mais eficiente para acumular carbono orgânico nos $20 \mathrm{~cm}$ superiores do solo, e o acúmulo diminuiu de 20 a $100 \mathrm{~cm}$; sistemas de cacau com idade superior a 20 anos foram semelhantes à floresta natural em relação ao acúmulo de carbono orgânico ao longo do perfil do solo (Monroe et al., 2016).

Portanto, os resultados indicam que a exportação de nutrientes e a imobilização destes na biomassa do sistema agroflorestal alterou a fertilidade do solo, havendo necessidade de enriquecimento do arranjo e adubação orgânica ou mineral suplementar para manutenção da produtividade desses sistemas ao longo do tempo.

\section{Conclusões}

Os valores de cálcio, magnésio, potássio, soma de bases e CTC diminuem em sistemas agroflorestais com castanheira, cupuaçuzeiro e pupunheira, considerando as épocas iniciais de estabelecimento e o período de vintes anos após a implantação.

Há maior concentração de nutrientes e de carbono orgânico na camada de $0-20 \mathrm{~cm}$ dos sistemas agroflorestais avaliados, em relação as profundidades de $20-40 \mathrm{~cm}$ e de $40-60 \mathrm{~cm}$.

Há tendência de redução de carbono orgânico total nos sistemas agroflorestais avaliados.

Os valores de cálcio, magnésio, potássio, soma de bases e CTC diminuem em sistemas agroflorestais com castanheira, cupuaçuzeiro e pupunheira, considerando as épocas iniciais de estabelecimento e o período de vintes anos após a implantação.

Há maior concentração de nutrientes e de carbono orgânico na camada de $0-20 \mathrm{~cm}$ dos sistemas agroflorestais avaliados, em relação as profundidades de $20-40 \mathrm{~cm}$ e de $40-60 \mathrm{~cm}$.

Há tendência de redução de carbono orgânico total nos sistemas agroflorestais avaliados.

\section{Referências}

Amaral EF, BROWN I F, MELOWF (2001) Efeito de diferentes usos da terra nas características do solo no Estado do Acre. Rio Branco: Embrapa Acre (Boletim de Pesquisa, 30). 20p.

Amaral EF, Melo AWF, Oliveira TK (2000) Levantamento de reconhecimento de baixa intensidade dos solos da região de inserção do projeto reca, estados de Rondônia, Acre e Amazonas. Embrapa Acre (Boletim de Pesquisa, 27). 40p.
Ayres MIC, Alfaia SS (2007) Calagem e adubação potássica na produção do cupuaçuzeiro em sistemas agroflorestais da Amazônia Ocidental. PesquisaAgropecuáriaBrasileira42(7):957-963.

Bartlett MS(1937) Properties of sufficiency and statistical tests. Proceedings of the Royal Society of London160:268-282.

Caetano JO, Verginassi A, Assis PCR, Carneiro MAC, Paulino HB (2013) Indicadores de qualidade de um latossolo vermelho sob diferentes sistemas de uso e manejo. Global Science and Technology 6(1).

Campos HF (2015) Solos e nutrição de plantas com foco em sistemas agroflorestais. In: Ciro AR, Marcos SB (ed) Sistemas Agroflorestais, Esalq. USP. p.35-5.

Cardinael R, Chevallier T, Barthès BG, Saby NPA, Parent T, Dupraz C, Bernoux M, Chenu C (2015) Impact of alley cropping agroforestry on stocks, forms and spatial distribution of soil organic carbon - A case study in a Mediterranean context. Geoderma 259-260:288299. doi: 10.1016/j.geoderma.2015.06.015.

Cerda R, Avelino J, Harvey CA, Gary C, Tixier P, Allinne C (2020) Coffee agroforestry systems capable of reducing disease-induced yield and economic losses while providing multiple ecosystem services. Crop Protection 134:105149. 10.1016/j.cropro.2020.105149.

Chen C, Liu W, Jiang X, Wu J (2017) Effects of rubberbased agroforestry systems on soil aggregation and associated soil organic carbon: Implications for land use. Geoderma 299:13-24. doi: 10.1016/j.geoderma.2017.03.021.

Defelipo BV, Ribeiro AC (1997) Análise química do solo. 2ed. UFV26p.

EMBRAPA (2009) Empresa Brasileira de Pesquisa Agropecuária. Manual de métodos de análise de solo. 2 ed. Embrapa Solos, 623p.

Fearnside PM (1989) Agricultura na Amazônia. Tipos de agricultura: padrão e tendências. In: Castro EM, Hebette J Na trilha dos grandes projetos. Modernização e Conflito na Amazônia. UFPA/NAEA. Caderno NAEA, 10.

Fernandes ECM, Biot Y, Castilla C, Cant A, Matos JCS, Garcia S, Perin R, Wandelli E (1997) The impact of selective logging and forest conversion for subsistence agriculture and pastures on terrestrial nutrient dynamics in the Amazon. Ciencia e Cultura Journal of the Brazilian Association for the Advancement of Science (49):34-47.

Guo J, Wang B, Wang G, Myo STZ, Cao F (2020) Effects of three cropland afforestation practices on the vertical distribution of soil organic carbon pools and nutrients in eastern China. Global Ecology and Conservation 22: e00913. doi: 10.1016/j.gecco.2020.e00913. 
Iwata BF, Leite LFC, Araújo ASF, Nunes LAPL, Gehring C, Campos LP (2012) Sistemas agroflorestais e seus efeitos sobre os atributos químicos em Argissolo Vermelho-Amarelo do Cerrado piauiense. Revista Brasileira de Engenharia Agrícola e Ambiental, 16(7):730-738.

Leite LFC, Galvão SRS, Holanda Neto MR, Araújo FS, Iwata BF (2010) Atributos químicos e estoques de carbono em Latossolo sob plantio direto no cerrado do Piauí. Revista Brasileira de Engenharia Agrícola e Ambiental14(12):1273-1280.

Liao JD, Boutton TW, (2008) Soil microbial biomass response towoodyplantinvasionofgrassland. Soil Biol. Biochemistry40:1207-1216. 1016/j.soilbio.2007.12.018. doi:10.

Lima SS, Leite LFC, Oliveira FC, Costa DB (2011) Atributos químicos e estoque de carbono e nitrogênio em Argissolo Vermelho-Amarelo sob sistemas agroflorestais e agricultura de corte e queima no norte do Piauí. RevistaÁrvore35(1):51-60.

Luizão FJ, Fearnside PM, Cerri CEP, Lehmann J (2009) The maintenance of soil fertility in Amazonian managed systems. In: Keller M, Bustamante M, Gash J, Silva Dias P.(eds.). Amazonia and Global Change.Geophysical Monograph Series.American Geophysical Union (AGU), 186:311-336.

Mcgrath D, Duryea ML, Cropper WP (2001) Soil phosphorus availability and fine root proliferation in Amazonian agroforests 6 years follwing forest conservation. Agriculture83(3):271-284.

Moline EFV,Coutinho ELM (2015) Atributos químicos de solos da Amazônia Ocidental após sucessão da mata nativa em áreas de cultivo. Revista de Ciências Agrárias/AmazonianJournalofAgriculturaland Environmental Sciences58(1):14-20.

Monroe PHM, Gama-Rodriguesb EF,GamaRodriguesb AC, Marquesc JRB (2016) Soil carbon stocks and origin under different cacao agroforestry systems in Southern Bahia, Brazil. Agriculture, Ecosystems and Environment 221:99-108. doi: 10.1016/j.agee.2016.01.022.

Nijmeijer A, Lauri P-E, Harmandd J-M, Freschet GT, Nieboukaho J-D E, Fogang PK, Enock S, Saj S (2019) Long-term dynamics of cocoa agroforestry systems established on lands previously occupied by savannah or forests. Agriculture, Ecosystems and Environment 275: 100-111. doi: 10.1016/j.agee.2019.02.004.

Redin M, Santos GF, Miguel P, Denega GL, lupatini M, Doneda A, Souza EL (2011) Impactos da queima sobre atributos químicos, físicos e biológicos do solo. Ciência Florestal 21:381-392.

Ribeiro JM, Frazão LA, Cardoso PHS, Oliveira ALG, Sampaio RA, Fernandes LA(2019) Fertilidade do solo e estoques de carbono e nitrogênio sob sistemas agroflorestais no Cerrado Mineiro. Revista Ciência Florestal 29(2):913-923. doi: 10.5902/1980509825310
Oliveira NA, Oliveira NA, Silva KR, Silva LJA, Melo AH (2017) Atributos químicos de solo sob diferentes sistemas de uso e manejo no projeto de assentamento Veneza - são domingos do Araguaia, Pa. Revista Agroecossistemas9(1):170-179.

Oliveira NL, Clara J, Mauricio D, Florian D (2010) Desenvolvimento Sustentável e Sistemas Agroflorestais na Amazônia Matogrossense. CONFINS: Revista Científica Franco Brasileira de Geografia 10. doi: 10.4000/confins.6778.

Reyna-Bowen L, Fernandez-Rebollo P, FernándezHabas J, Gómez JÁ (2020) The influence of tree and soil management on soil organic carbon stock and pools in dehesa systems. Catena190:IF 3.851. doi: 10.1016/j.catena.2020.104511.

Sá IB, Cunha TJF, Teixeira AHC, Angelotti F, Drumond FM (2010) Desertificação no Semiárido brasileiro. In: ICID+18 Conferência Internacional: Clima, Sustentabilidade e Desenvolvimento em Regiões Semiáridas, 2., Resumos... Fortaleza.

Sedam (2012) Secretaria de Estado do Desenvolvimento Ambiental. Climatologia do Estado de Rondônia. Disponível em: <http://www.sedam.ro.gov.br/web/guest/Meteorologia/ Climatologia/>. (Acesso em 27 out 2014).

Shapiro SS, Wilk MB (1965) An analysis of variance test for normality (complete samples. Biometrika, Boston 52(3-4):591-611.

Silva DC, Silva M L N, Curi N, Oliveiras A H, Souza F S, Martins S G, Macedo R L G (2011) Atributos do solo em sistemas agroflorestais, cultivo convencional e floresta nativa. Revista de Estudos Ambientais 13(1):77-86.

Silva, FAS (2013) ASSISTAT: Assistência Estatística (versão 7.7). Universidade Federal de Campina Grande. Campina Grande. PB. Disponível em: www.assistat.com/indexp.html. (Acesso em $05 \mathrm{dez}$ 2014).

Tukey J.W (1949) Comparing individual means in the analysis of variance. Biometrics, Washington 5(2):99-114 .

Tumwebaze SB, Byakagaba P (2016) Soil organic carbon stocks under coffee agroforestry systems and coffee monoculture in Uganda. Agriculture, Ecosystems and Environment 216: 188-193. doi: 10.1016/j.agee.2015.09.037.

Walkley A, Black I A (1934) An examination of the Degtjareff method for determining soil organic matter and a proposed modification of the chromic acid titration method. Soil Science 37:29-38.

Wu J, Zeng H, Zhao F, Chen C, Liu W, Yang B, Zhang W (2020) Recognizing the role of plant species composition in the modification of soil nutrients and water in rubber agroforestry systems. Science of The Total Environment 723:138042. doi: 10.1016/j.scitotenv.2020.138042. 